\title{
Quantitative Models for Describing Temperature and Moisture Effects on Sporulation of Phomopsis amygdali on Peach
}

\author{
N. Lalancette, K. A. Foster, and D. M. Robison
}

Rutgers University, Agricultural Research and Extension Center, 121 Northville Road, Bridgeton, NJ 08302. Current address of D. M. Robison: 617 Marple Ave., Canonsburg, PA 15317. Accepted for publication 14 April 2003.

\begin{abstract}
Lalancette, N., Foster, K. A., and Robison, D. M. 2003. Quantitative models for describing temperature and moisture effects on sporulation of Phomopsis amygdali on peach. Phytopathology 93:1165-1172.

Twigs with constriction cankers were pruned from a Prunus persica 'Jerseyglo' orchard and placed in incubators under high humidity ( $>95 \%)$ at constant temperatures of $-6,1,10,17,24,31,38$, and $45^{\circ} \mathrm{C}$. Cankers were removed for observation after $2,6,24,48,72$, and $96 \mathrm{~h}$ incubation. Sporulation was expressed as the percentage of pycnidia producing cirri and the number of conidia per pycnidium or canker. The experiment was first performed during 1998 to 1999 and then repeated in 1999 to 2000 and 2000 to 2001. Sporulation was modeled by fitting the Richards function to each dependent variable with duration of high relative humid-

Richards model were expressed as Gaussian and quadratic functions of temperature, respectively. Models created from the first two experimental repetitions were validated by statistical comparison to those created independently from the third repetition. When models were fit to the pooled data, temperature and moisture described 69 to $80 \%$ of the variation in sporulation. The models specified a temperature ranging from 0 to $37^{\circ} \mathrm{C}$ (optimum temperatures for cirri formation and conidia production range from 19 to 20 and 22 to $23^{\circ} \mathrm{C}$, respectively), and the majority of sporulation occurred between 16 and $48 \mathrm{~h}$ from initiation of the high humidity period. These results show that the environmental criteria for sporulation coincide with those that prolong the susceptibility of infection courts during fall and spring.
\end{abstract} ity as the independent variable. The asymptote and rate parameters of the

The sporulation process by which a fungal plant pathogen produces inoculum for infection can be divided into three distinct phases: production of sporophores, production of spores, and maturation of spores (9). In constriction canker of peach, caused by Phomopsis amygdali (Del.) Tuset \& Portilla (syn. Fusicoccum amygdali Del.), the first stage consists of the formation of erumpent, black pycnidia on twig cankers (8). The length of the latent period, the time from infection to formation of mature pycnidia, has not been quantitatively determined for P. amygdali. However, early field and greenhouse studies have indicated that the length of the incubation period, the time from infection to first canker symptoms, is approximately 1 month $(2,7)$. None of these initial studies examined the production and viability of pycnidia over time, two important factors that determine the amount of inoculum available for infection.

Research on elucidating the disease cycle indicated that the pathogen infects twigs during the fall through leaf scars, and in the spring through bud scale scars or flowers (2). These results supported earlier observations that pycnidia were present throughout the growing season in orchards having constriction canker $(5,6)$. That is, infection during these two seasons resulted in adjoining and sometimes overlapping cohorts of pycnidia. Furthermore, a recent quantitative study revealed that mean canker size and number of pycnidia per canker followed sinusoidal patterns, with minimum and maximum amplitudes in the spring and fall, respectively (15). However, regardless of this pattern, the results also revealed that mature pycnidia capable of producing significant numbers of conidia ( $10^{6}$ to $10^{7}$ per canker) were present throughout the year; that is, considerable spore production occurred even when amplitudes were at a minimum. Thus, it was postulated that

Corresponding author: N. Lalancette; E-mail address: lalancette@aesop.rutgers.edu

Publication no. P-2003-0711-01R

(C) 2003 The American Phytopathological Society
Additional keywords: Fusicoccum canker, monocycle.
$P$. amygdali acts as an $r$ strategist by maintaining a high level of sporulating potential at all times $(15,23)$.

Given that viable pycnidia are present throughout the year, the occurrence of the second phase of the sporulation process (production of spores) determines if and when inoculum will become available for infection. $P$. amygdali pycnidia sporulate by exuding spores through their ostioles as either dull white masses or long cirri $(5,19)$. The vast majority of these asexual spores are the fusoid $\alpha$ conidia, whereas the filiform $\beta$ conidia are rare (21). A number of studies have shown that this sporulation process is dependent on two abiotic environmental factors, moisture and temperature. Field observations of cirri were often made following wet weather (8), and pycnidia on detached cankers were observed to sporulate when placed in a moist chamber (19). When cankers were placed under moist conditions at three different constant temperatures, the number of pycnidia observed to sporulate was dependent on temperature (7). Finally, a recent field study showed that production and dispersal of conidia was favored by moderate temperatures and high rainfall (22).

Constriction canker is known to occur on peach, almond, and plum (24). Currently, no data are available on any of these suscepts that describe the combined effects of temperature and moisture on the production and release of spores by $P$. amygdali. Specifically, the optimum environmental conditions that favor maximum sporulation and the extreme conditions or bounds that limit sporulation are not known. Thus, the main objective of this study was to quantitatively describe the sporulation process over the entire range of applicable environmental conditions. Mathematical models defining a response surface will be developed to provide a visualization of the interacting environmental effects as well as descriptive parameters. Given prior observations on the effects of environment $(7,19)$, pycnidia sporulation and conidia production will be expressed as a function of temperature and duration of high relative humidity. And because free moisture, often in the form of rainfall, has been shown to be important for initiation of sporu- 
lation $(8,22)$, the experimental cankers will begin their incubation with a wetting period. Such basic descriptive information on sporulation will be useful for understanding the epidemiology of constriction canker, particularly with respect to inoculum availability during infection periods in the fall and spring. Partial results of this study were reported previously (12).

\section{MATERIALS AND METHODS}

Canker samples. Twigs bearing constriction cankers were pruned from an 8-year-old 'Jerseyglo' peach block grafted on 'Lovell' rootstock. Only mature 6- to 12-month-old cankers bearing abundant pycnidia were selected; canker length ranged from approximately 2 to $5 \mathrm{~cm}$. To minimize canker desiccation, twigs were pruned so that at least $5 \mathrm{~cm}$ of noninfected tissue bordered each side of the canker. Canker samples were harvested at regular intervals from October through April. Each sample consisted of 30 cankers arbitrarily collected from trees throughout the block. Samples were stored dry in a plastic bag at room temperature for a maximum of $24 \mathrm{~h}$ prior to treatment. The experiment was first performed from fall to spring of 1998 to 1999 (rep 1) and then repeated in 1999 to 2000 (rep 2) and 2000 to 2001 (rep 3).

Incubation treatments. Each sample of 30 cankers was soaked for $5 \mathrm{~min}$ in deionized water at a specified temperature and patted dry with paper towels. The cankers were then arbitrarily divided into 10 subsamples, each containing three cankers. Subsamples were placed in plastic trays $(18 \times 14 \times 3 \mathrm{~cm}$ deep $)$ maintained at high relative humidity $(>95 \%)$ by lining the bottom with moist blotting paper and covering the tray with a tight-fitting lid. All 10 trays were placed in an incubator for a specified duration at a designated constant temperature. Thus, a total of 30 cankers (10 trays at three cankers per tray) were incubated and assessed at each treatment temperature and duration of high relative humidity. Trays were checked for moisture content every $24 \mathrm{~h}$, and water was added as needed to maintain relative humidity above $95 \%$.

Incubation treatments consisted of eight temperatures: $-6,1,10$, $17,24,31,38$, and $45^{\circ} \mathrm{C}$. For temperatures of 1 to $38^{\circ} \mathrm{C}$, cankers were assessed for sporulation at durations of 2, 6, 24, 48, 72, and $96 \mathrm{~h}$; at -6 and $45^{\circ} \mathrm{C}$, assessments were only conducted at 2,48 , and $96 \mathrm{~h}$. Thus, a total of 42 treatments ( 6 temperatures $\times 6$ durations +2 temperatures $\times 3$ durations) and 1,260 cankers (42 treatments $\times 30$ cankers per treatment) were examined for each of the three experimental repetitions. The order of examination of each treatment within each repetition was chosen randomly.

Temperature during incubation periods was recorded electronically using a portable Hobo datalogger (Onset Computer Corp., Bourne, MA). Accuracy of this instrument was checked prior to and after each replication at five temperature levels using a NIST (National Institute of Standards and Technology)-certified mercury thermometer. Any temperature deviations from this standard were corrected based on the generated calibration curve.

Sporulation assessment. After completion of the designated incubation period, the total number of pycnidia and sporulating pycnidia were counted for each of the 10 subsamples with a dissecting microscope at low power. A pycnidium was considered to be sporulating if any part of a cirrus could be observed emanating from its ostiole. After counting pycnidia, cankers from each subsample were washed with water using a DeVilbiss atomizer set at 5 psi (DeVilbiss Health Care, Inc., Somerset, PA). Conidia from each subsample of three cankers were bulk collected in a flask to which 1 to 2 drops of a dilute Tween 20 solution ( 1 drop of Tween 20 per liter of water) were added. After agitation, the concentration of conidia in the suspension was estimated by averaging 10 hemacytometer chamber counts performed with the aid of a compound microscope $(\times 200)$. Total number of conidia produced per subsample was determined from this concentration and the total volume of the suspension. Only $\alpha$ conidia were counted in the assessment; $\beta$ conidia were rarely observed and are not considered germinable. From these data, three dependent variables were calculated for each of the 10 subsamples in each treatment: S1 = percentage of sporulating pycnidia; $\mathrm{S} 2=$ number of conidia per pycnidium; and S3 = number of conidia per canker.

Model development. The procedure used for modeling sporulation as a function of moisture and temperature consisted of five interrelated steps. First, the upper asymptote of each sporulationdependent variable needed to be estimated from the data. This task was accomplished by searching for the maximum mean value across all durations of incubation within each temperature treatment. These means served as constants when estimating other parameters during subsequent intermediate operations.

In the second step, the linearized version of the Richards model, chosen for its flexibility in conforming to different patterns of growth (18), was fit to the data for each temperature:

$$
\ln \left\{1 /\left[1(S / K)^{1-m}\right]\right\}=r H
$$

in which $S$ is the dependent sporulation variable ( $\mathrm{S} 1, \mathrm{~S} 2$, or $\mathrm{S} 3$ ), $K$ is the estimated asymptote from step one, $m$ is the shape parameter, $r$ is the rate parameter, and $H$ is the duration of relative humidity $>95 \%$. A no-intercept version was fit because zero sporulation occurred at $H=0 \mathrm{~h}$ (i.e., no cirri were present at initiation of incubation). To avoid division by zero, small constants of 1,10 , and 100 were added to $K$ for variables $\mathrm{S} 1, \mathrm{~S} 2$, and $\mathrm{S} 3$, respectively. Because the Richards model can emulate growth patterns ranging from the monomolecular at $m=0$ to the logistic at $m=2$, an iterative approach was used to search for the best value of the shape parameter. At each temperature level, separate regressions were performed for values of $m$ ranging from 0 to 2 by 0.1 increments. The regression model was considered to have the best fit of the data when the value of $m$ yielded the highest coefficients of determination across all temperature levels.

In the third development step, the rate parameter estimates from equation 1 were plotted against temperature. Visual examination of these graphs, created for each experimental repetition of each dependent variable, indicated the existence of a parabolic relationship. Thus, the parameter estimates were regressed against temperature $(T)$ by fitting a quadratic model:

$$
r=b_{0}+b_{1} T+b_{2} T^{2}
$$

where $r$ is the Richards model rate parameter estimates $r_{1}, r_{2}$, and $r_{3}$ for each dependent variable $\mathrm{S} 1, \mathrm{~S} 2$, and $\mathrm{S} 3$, respectively. This step confirmed the relationship between the rate parameter and temperature.

In the fourth step, $S$ was modeled as a function of $T$ and $H$ by substituting the rate parameter function (equation 2) for $r$ in the Richards model (equation 1) to yield

$$
\ln \left\{1 /\left[1-(S / K)^{1-m}\right]\right\}=b_{1} H+b_{2} H T+b_{3} H T^{2}
$$

The coefficients of this function were estimated for each of the three experimental repetitions using the calculated mean values of each dependent variable. As in equation $1, K$ was set equal to the asymptote values derived in step one; to avoid division by zero, small constants of 1, 10, and 100 were added to $K$ for variables S1, S2, and $\mathrm{S} 3$, respectively. Similarly, a range of shape parameter values $(0 \leq$ $m \leq 2$ ) was iteratively examined to search for the best overall fit.

Since $K$ is a constant in equation 3 (for each temperature level), the fifth and final step involved modeling this upper asymptote as a function of temperature $(T)$. The maximum mean sporulation values estimated in step one were plotted against their respective temperatures. The resulting curves displayed optimum, maximum, and minimum values and had shapes similar to that of a normal distribution. Thus, the relationship between $K$ and $T$ was modeled by fitting a generalized form of the Gaussian function:

$$
K=a \exp \left[-s(T-t)^{2}\right]
$$

where $a, s$, and $t$ describe the curve's amplitude, spread, and location of its maximum, respectively. In terms of pathogen biol- 
ogy, $a$ is the maximum amount of sporulation, $s$ determines the temperature range over which sporulation occurs (spread between the minimum and maximum temperatures), and $t$ is the optimum temperature for sporulation. Together, this equation and the nonlinear form of equation 3 constitute the final model for predicting sporulation as a function of temperature and duration of high relative humidity. One set of equations 3 and 4 were created for each dependent variable.

Statistical analysis. All equations in model development steps one through four were fit to data using the linear regression procedure of the Statistical Analysis System version 8.2 (SAS Institute, Cary, NC). The Gaussian model in step five was fit using nonlinear regression analysis (NLIN procedure). Each regression analysis was performed on the means of the 10 subsample data values.

Goodness-of-fit of model equations 2 and 3 was evaluated by examination of the coefficients of determination and the standard errors of the parameter estimates. However, when fitting equation 1 to the data, the sum of the $R^{2}$ values of the regressions performed across all temperature levels was used to determine which value of $m$ provided the best overall fit. Aptness of nonlinear equation 4 was ascertained by examining the coefficients of variation of the parameter estimates and by graphical comparison of predicted and observed values.

Models for describing each dependent variable were developed from the pooled experimental repetition 1 and 2 (rep 1+2) data sets. Separate analyses were performed on the repetition 3 data to allow creation of an independent model for validation. Full and reduced models were compared using an $F$ test to determine if the estimated parameter coefficients from the repetition 3 models were statistically identical to those derived from the rep $1+2$ data (17). If the independently derived models were found to be equivalent, then the data from all experimental repetitions can be pooled to allow for increased precision.

\section{RESULTS}

Treatment effects. Sporulation of pycnidia (S1) was observed between 1 and $38^{\circ} \mathrm{C}$, with little or no visible cirri formation occurring at temperature extremes of -6 and $45^{\circ} \mathrm{C}$ (Fig. 1A). The greatest proportion of pycnidia produced cirri at $17^{\circ} \mathrm{C}$, reaching maximum proportions after 72 to $96 \mathrm{~h}$ incubation. As expected, conidia production, whether measured as conidia per pycnidium or conidia per canker, occurred over the same 1 to $38^{\circ} \mathrm{C}$ temperature range as observed for cirri formation (Fig. 1B and C). However, maximum conidia production occurred at a warmer temperature of $24^{\circ} \mathrm{C}$. This requirement of higher temperatures for conidia production was particularly evident when comparing the 10 and $31^{\circ} \mathrm{C}$ treatments. Although the percentage of pycnidia forming cirri at these two temperatures was very similar at all incubation durations (Fig. 1A), conidia production was considerably greater at the higher temperature (Fig. 1B and C). For example, after $96 \mathrm{~h}$ incubation, the number of conidia per pycnidium was approximately five times greater at $31^{\circ} \mathrm{C}$ than at $10^{\circ} \mathrm{C}$.

Maximum conidia production levels agreed with dissecting microscope observations on cirri development. In addition to increasing percentages of sporulating pycnidia, cirri growth also appeared to contribute to conidia production. After the same duration of incubation, cirri length was noticeably longer at more favorable temperatures than at less favorable temperatures. For example, at any given time of incubation, cirri formed at $31^{\circ} \mathrm{C}$ were observed to be longer than those formed at $10^{\circ} \mathrm{C}$. Thus, if conidia numbers increase with increasing cirri length, then their growth may also contribute to conidia production.

The number of pycnidia per canker in harvested twig samples varied somewhat across the 3 years (experimental repetitions) of the study. When averaged across all treatment combinations, the mean number of pycnidia per canker $( \pm \mathrm{SE})$ was $394 \pm 4$ for 1998 to 1999 (rep 1), $583 \pm 10$ for 1999 to 2000 (rep 2), and $475 \pm 6$ for 2000 to 2001 (rep 3). Thus, pycnidial numbers in repetition 2 were $48 \%$ greater than those observed in repetition 1 and $23 \%$ greater than those observed in repetition 3 .
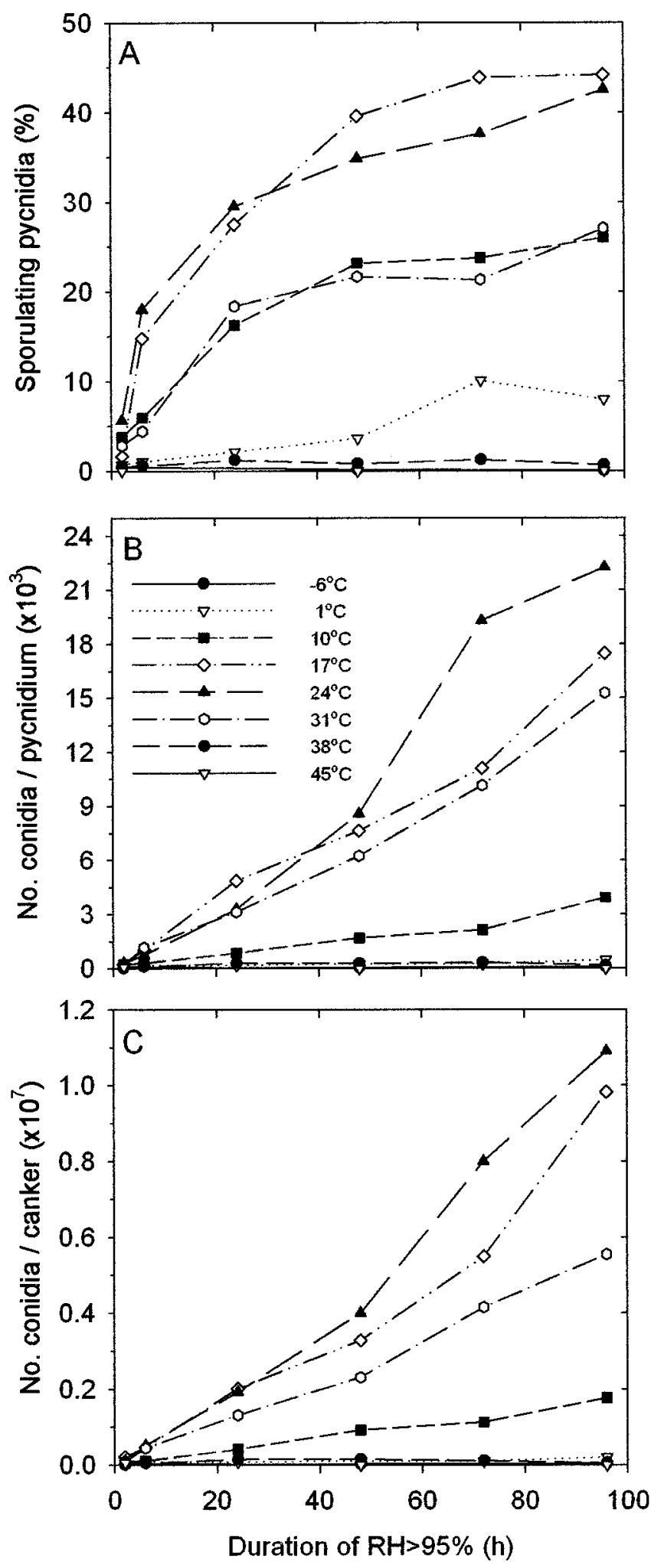

Fig. 1. Effect of temperature and duration of high relative humidity on sporulation of Phomopsis amygdali pycnidia on detached peach twig cankers. Sporulation was assessed as $\mathbf{A}$, the percentage of pycnidia producing visible cirri; $\mathbf{B}$, the quantity of conidia produced per pycnidium; and $\mathbf{C}$, the quantity of conidia produced per canker. Data are means of three experimental repetitions, each consisting of observations on 30 cankers. Conidia production was estimated with a hemacytometer. 
Estimation of $\boldsymbol{K}$. The upper asymptote, $K$, estimated by the maximum mean value of observed sporulation ( $S 1, S 2$, or S3), was attained after 72 to $96 \mathrm{~h}$ incubation for most temperature levels (Fig. 1). For the $\mathrm{S} 1$ dependent variable, maximum $K$ values of 58.0 and $21.8 \%$ sporulating pycnidia occurred for repetitions $1+2$ and 3 , respectively. These maxima occurred at $17^{\circ} \mathrm{C}$ for repetitions $1+2$, but at 17 and $24^{\circ} \mathrm{C}$ for repetition 3 . Based on graphical comparison of predicted and observed data, the Gaussian model provided a good to excellent fit to both data sets (Fig.
2A); parameter coefficients of variation also indicated a good model fit, ranging from 1.0 to $32.5 \%$ (Table 1). Maximum percentage of sporulating pycnidia, as estimated by the $a$ parameter, was considerably different for the rep $1+2$ and 3 data sets; however, the estimated optimum temperature ( $t$ parameter) was very similar at 19.2 and $20.2^{\circ} \mathrm{C}$, respectively.

The relationship of $K$ to temperature for the conidia production variables $\mathrm{S} 2$ and $\mathrm{S} 3$ followed a similar pattern to that observed for the S1 dependent variable. Graphical comparison of observed and
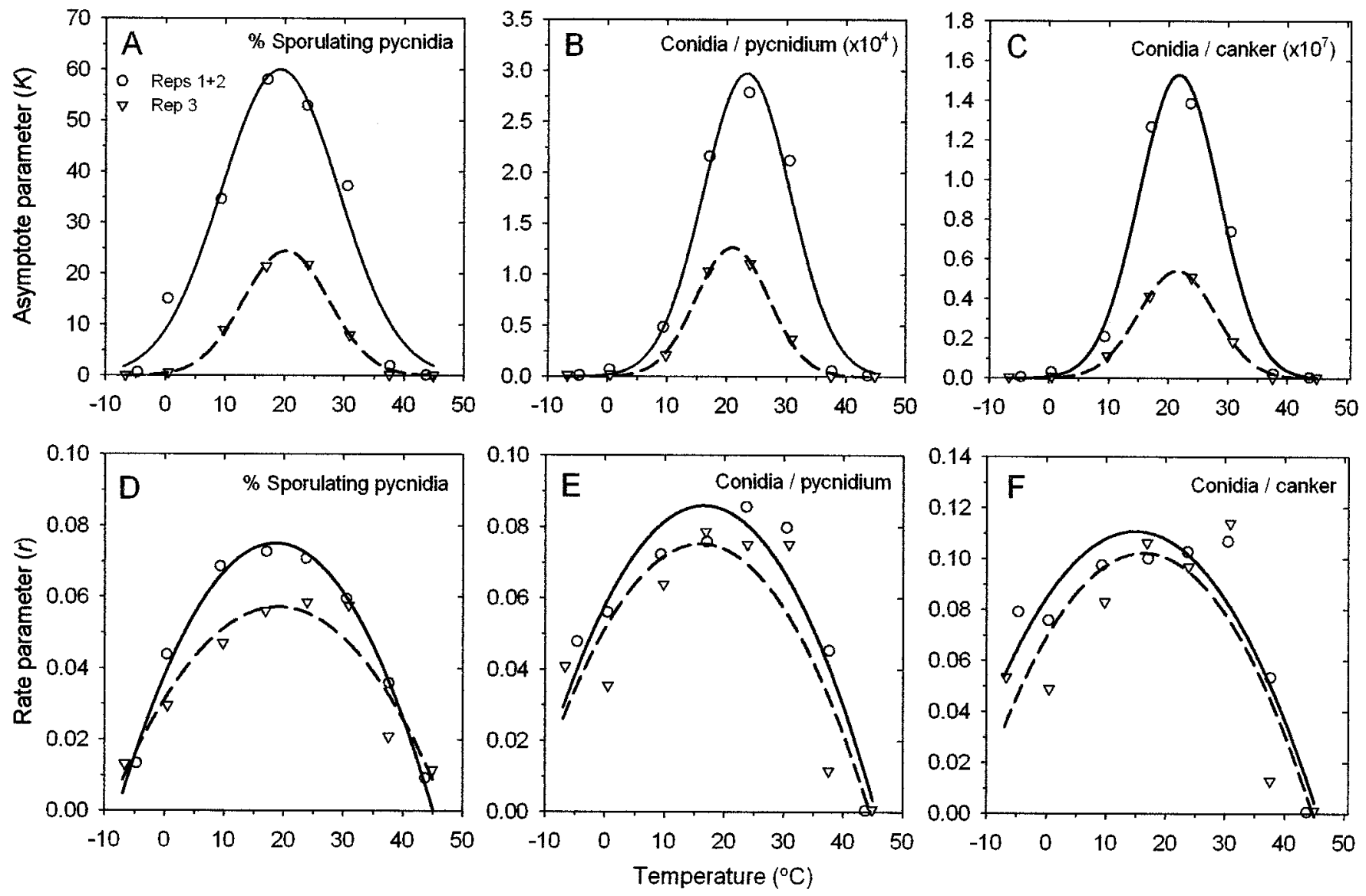

Fig. 2. Estimation of the asymptote parameter $(K)$ and the rate parameter $(r)$ of the Richards model as a function of temperature for three dependent variables describing sporulation of Phomopsis amygdali on peach. Data points for $K$ are maximum sporulation values observed at each temperature level; data points for $r$ are rate parameters estimated by fitting the Richards model to each sporulation curve at each temperature level (equation 1). Curves represent predicted parameter values for pooled experimental repetition 1 and 2 data (solid line) and repetition 3 data (dashed line). Predicted values in $\mathbf{A}, \mathbf{B}$, and $\mathbf{C}$ are based on fitting the Gaussian model (Table 1); predicted values in $\mathbf{D}, \mathbf{E}$, and $\mathbf{F}$ are based on fitting the quadratic model (Table 2).

TABLE 1. Estimation of the asymptote parameter $(K)$ for three dependent variables describing Phomopsis amygdali sporulation on peach twig cankers by fitting the Gaussian model to maximum sporulation observed at eight temperature levels

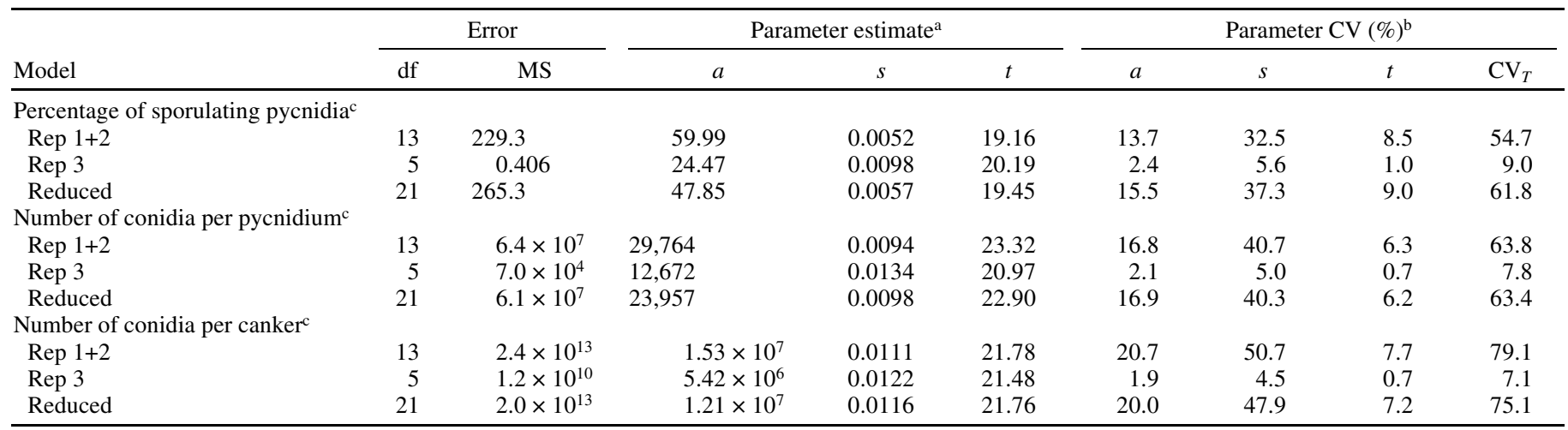

$\bar{a}$ Gaussian model (equation 4) fit to each data set using nonlinear regression. All analyses on experimental repetition (rep) or reduced data had significant $F$ values $(P<0.05)$.

${ }^{\mathrm{b}}$ Parameter coefficient of variation calculated as $\mathrm{CV}=(\mathrm{SE} / \mathrm{estimate}) \times 100 ; \mathrm{CV}_{T}$ is the total or sum of variation across all parameters.

${ }^{c}$ Data values for each dependent variable were calculated from 10 sets of three constriction cankers per treatment. Conidia production was estimated as a mean of 10 hemacytometer chamber counts per set, totaling 100 chamber counts per treatment. 
predicted data indicated that the Gaussian model provided a good fit (Fig. 2B and C). As with the S1 variable, observed maximum $K$ values and $a$ parameter estimates for the rep $1+2$ data were greater than for the rep 3 data (Table 1). However, estimated $t$ parameters were very similar for models created from each data set, particularly for the $\mathrm{S} 3$ variable. Based on the $t$ parameter estimates, optimum temperatures ranged from 21.0 to $23.3^{\circ} \mathrm{C}$.

Estimation of $r$. When fitting the Richards function (equation 1) to each temperature level, the highest $R^{2}$ sums for each combination of dependent variable and experimental repetition occurred when $m=0.9$. This value of the shape parameter defines a model with properties very similar to that of the Gompertz model. At this value of $m$, regressions at temperature levels of 1 to $38^{\circ} \mathrm{C}$ had mean $R^{2}$ values of $0.80,0.76$, and 0.70 for $\mathrm{S} 1, \mathrm{~S} 2$, and $\mathrm{S} 3$, respectively. Given the diminished sporulation response at temperature extremes of -6 and $45^{\circ} \mathrm{C}, R^{2}$ values for these levels were lower, with means of $0.40,0.48$, and 0.45 for S1, S2, and S3, respectively.

Examination of plots of predicted versus observed data revealed that the quadratic model provided good to excellent fits to the estimated rate parameters of the Richards function (Fig. 2D to F). Overall, temperature explained 61 to $89 \%$ of the variation in the rate parameter for the three dependent variables (Table 2). The curves generated for the $\mathrm{S} 1$ variable depicted minimum rates of sporulating pycnidia at each temperature extreme and a maximum near $17^{\circ} \mathrm{C}$ (Fig. 2D). However, observed rates for the conidia production S2 and S3 variables were somewhat less parabolic, being higher than the predicted rates at temperatures between 25 and $35^{\circ} \mathrm{C}$ (Fig. 2E and F). Also, relatively high rates were estimated by the Richards function for the -6 and $1^{\circ} \mathrm{C}$ levels. Because little sporulation was observed at these temperatures, this discrepancy was due to overestimation of $r$ by the Richards model. Essentially, the selected value of $m(0.9)$ was a compromise across all temperature levels; better fits at these levels may have been possible if $m$ itself was an estimated parameter.

Sporulation models. All regression models (equation 3) fitted to the rep $1+2$ and 3 data sets of each dependent variable had highly significant $F$ values and parameter estimates $(P<0.01)$. For most combinations of dependent variable and data set, temperature and duration of high relative humidity described 70 to $81 \%$ of the variation in sporulation (Table 3 ). Based on the coefficients of determination, the models quantitatively described the percentage of sporulating pycnidia best, although relatively good fits were observed for the number of conidia per pycnidium variable (Table 3 ). The coefficients of variation of the parameter estimates for both data sets ranged from 13 to $20 \%$, 18 to $31 \%$, and 12 to $38 \%$ for models fitted to the S1, S2, and S3 dependent variables, respectively.

TABLE 2. Estimation of the Richards model rate parameter $(r)$ for three dependent variables describing sporulation of Phomopsis amygdali on peach twig cankers by fitting quadratic functions of temperature

\begin{tabular}{|c|c|c|c|c|c|c|c|c|c|}
\hline \multirow[b]{2}{*}{ Model $^{\mathrm{a}}$} & \multicolumn{2}{|c|}{ Error } & \multicolumn{3}{|c|}{ Parameter estimate $^{b}$} & \multicolumn{3}{|c|}{ Parameter standard error ${ }^{b}$} & \multirow[b]{2}{*}{$R_{a}^{2}$} \\
\hline & $\mathrm{df}$ & MS & $b_{0}$ & $b_{1}$ & $b_{2}$ & $b_{0}$ & $b_{1}$ & $b_{2}$ & \\
\hline \multicolumn{10}{|c|}{ Percentage of sporulating pycnidia ${ }^{c}$} \\
\hline Rep $1+2$ & 13 & $7.2 \times 10^{-5}$ & 0.0380 & 0.00399 & $-1.07 \times 10^{-4}$ & 0.0035 & $3.8 \times 10^{-4}$ & $9.6 \times 10^{-6}$ & 0.89 \\
\hline Rep 3 & 5 & $6.1 \times 10^{-5}$ & 0.0312 & 0.00273 & $-7.17 \times 10^{-5}$ & 0.0043 & $4.5 \times 10^{-4}$ & $1.1 \times 10^{-5}$ & 0.85 \\
\hline Reduced & 21 & $9.5 \times 10^{-5}$ & 0.0361 & 0.00350 & $-9.41 \times 10^{-5}$ & 0.0032 & $3.5 \times 10^{-4}$ & $8.6 \times 10^{-6}$ & 0.84 \\
\hline \multicolumn{10}{|c|}{ Number of conidia per pycnidium ${ }^{c}$} \\
\hline Rep $1+2$ & 13 & $1.1 \times 10^{-4}$ & 0.0580 & 0.00338 & $-1.02 \times 10^{-4}$ & 0.0043 & $4.8 \times 10^{-4}$ & $1.2 \times 10^{-5}$ & 0.85 \\
\hline Rep 3 & 5 & $2.9 \times 10^{-4}$ & 0.0514 & 0.00297 & $-9.25 \times 10^{-5}$ & 0.0094 & $9.7 \times 10^{-4}$ & $2.4 \times 10^{-5}$ & 0.69 \\
\hline Reduced & 21 & $1.5 \times 10^{-4}$ & 0.0559 & 0.00324 & $-9.90 \times 10^{-5}$ & 0.0041 & $4.4 \times 10^{-4}$ & $1.1 \times 10^{-5}$ & 0.80 \\
\hline \multicolumn{10}{|c|}{ Number of conidia per canker ${ }^{c}$} \\
\hline Rep $1+2$ & 13 & $2.2 \times 10^{-4}$ & 0.0844 & 0.00353 & $-1.18 \times 10^{-4}$ & 0.0060 & $6.7 \times 10^{-4}$ & $1.6 \times 10^{-5}$ & 0.82 \\
\hline Rep 3 & 5 & $7.1 \times 10^{-4}$ & 0.0684 & 0.00411 & $-1.26 \times 10^{-4}$ & 0.0148 & 0.0015 & $3.8 \times 10^{-5}$ & 0.61 \\
\hline Reduced & 21 & $3.3 \times 10^{-4}$ & 0.0788 & 0.00378 & $-1.22 \times 10^{-4}$ & 0.0060 & $6.5 \times 10^{-4}$ & $1.6 \times 10^{-5}$ & 0.76 \\
\hline
\end{tabular}

${ }^{a}$ Quadratic model (equation 2) fit to data using linear regression analysis. $R_{a}{ }^{2}$ is the coefficient of determination adjusted for the number of independent variables.

b All regression analyses had significant $F$ values and parameter estimates $(P<0.05)$.

${ }^{\mathrm{c}}$ Data for the dependent variables consisted of $r$ parameter values generated from fitting the Richards model $(m=0.9)$ to sporulation data at each temperature (equation 1).

TABLE 3. Regression analysis results from modeling sporulation of Phomopsis amygdali on peach twig cankers as a function of temperature and duration high relative humidity for three different dependent variables

\begin{tabular}{|c|c|c|c|c|c|c|c|c|c|}
\hline \multirow[b]{2}{*}{ Model $^{\mathrm{a}}$} & \multicolumn{2}{|c|}{ Error } & \multicolumn{3}{|c|}{ Parameter estimate ${ }^{b}$} & \multicolumn{3}{|c|}{ Parameter standard error ${ }^{\mathrm{b}}$} & \multirow[b]{2}{*}{$R_{a}^{2}$} \\
\hline & $\mathrm{df}$ & MS & $b_{1}$ & $b_{2}$ & $b_{3}$ & $b_{1}$ & $b_{2}$ & $b_{3}$ & \\
\hline \multicolumn{10}{|c|}{ Percentage of sporulating pycnidia $^{\mathrm{c}}$} \\
\hline Rep 1+2 & 81 & 2.20 & 0.0387 & 0.0039 & -0.000106 & 0.0052 & 0.00057 & 0.000014 & 0.80 \\
\hline Reduced & 123 & 1.95 & 0.0363 & 0.0035 & -0.000095 & 0.0039 & 0.00042 & 0.000010 & 0.80 \\
\hline \multicolumn{10}{|c|}{ Number of conidia per pycnidium ${ }^{c}$} \\
\hline Rep 1+2 & 81 & 3.40 & 0.0565 & 0.0034 & -0.000101 & 0.0064 & 0.00071 & 0.000018 & 0.79 \\
\hline $\operatorname{Rep} 1+2$ & 81 & 8.26 & 0.0819 & 0.0037 & -0.000118 & 0.0100 & 0.00111 & 0.000027 & 0.72 \\
\hline Rep 3 & 39 & 11.31 & 0.0652 & 0.0045 & -0.000133 & 0.0158 & 0.00165 & 0.000041 & 0.59 \\
\hline Reduced & 123 & 9.12 & 0.0760 & 0.0040 & -0.000125 & 0.0084 & 0.00092 & 0.000023 & 0.69 \\
\hline
\end{tabular}

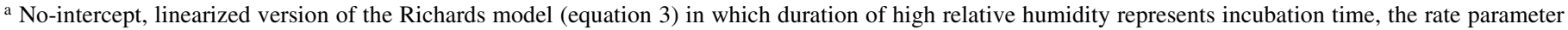

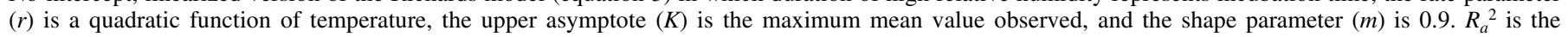
coefficient of determination adjusted for the number of independent variables.

b All regression analyses had significant $F$ values and parameter estimates $(P<0.01)$.

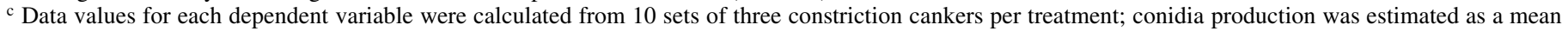
of 10 hemacytometer chamber counts per set, totaling 100 chamber counts per treatment. 
Model validation. Full and reduced models fitted to the rep $1+2$ and 3 data sets were statistically compared at each step of the development process. Comparison of the Gaussian models (equation 4) used to estimate the asymptote parameter $K$ resulted in $F$ values of $5.21(P=0.01), 3.19(P=0.05)$, and $2.22(P=0.12)$ for the S1, S2, and S3 variables, respectively. Hence, the model parameters generated from the rep 3 data for the S2 and S3 conidia production variables were not statistically different from those estimated from the rep $1+2$ data, thereby validating the
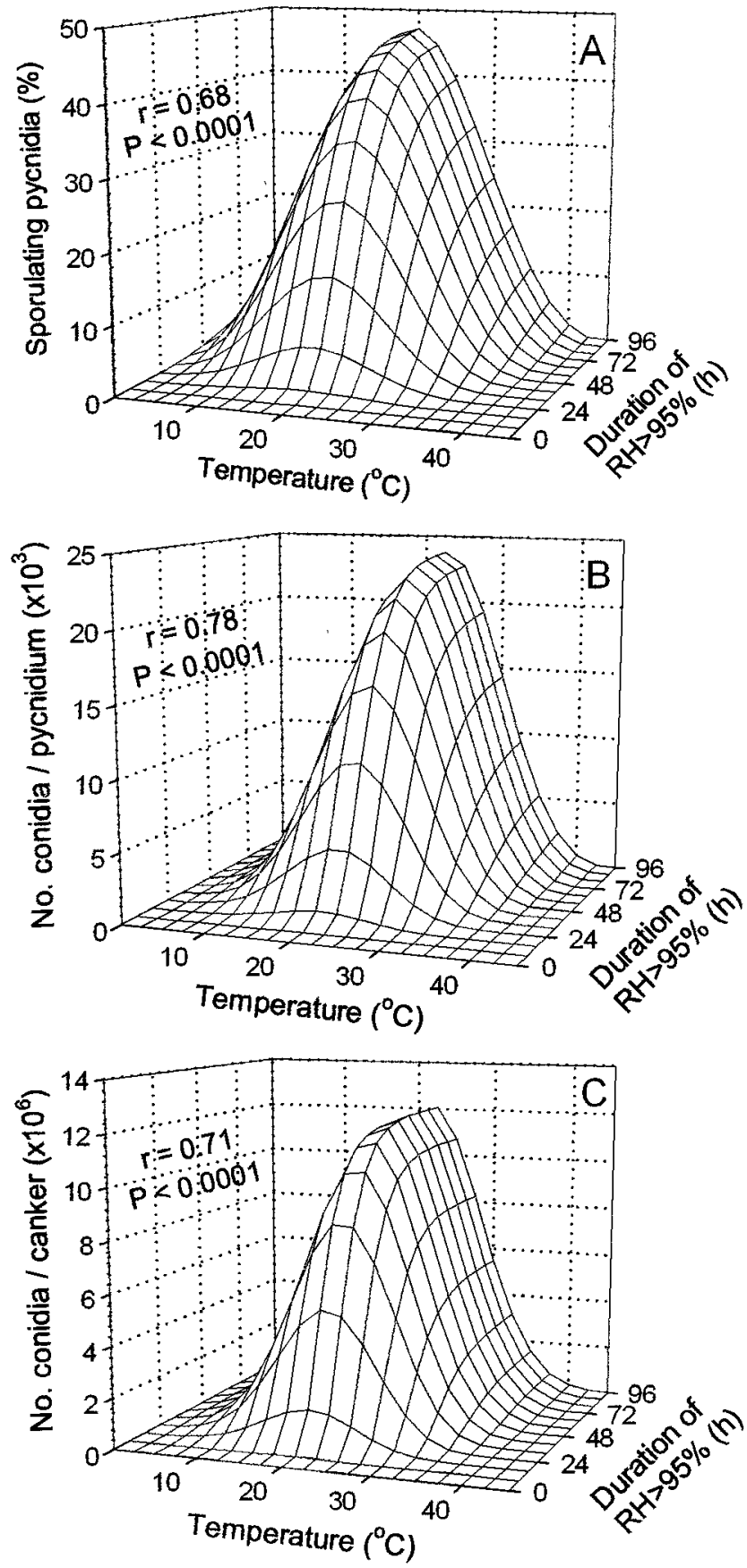

Fig. 3. Three-dimensional representations depicting sporulation of Phomopsis amygdali on peach as a function of temperature and duration of high relative humidity for $\mathbf{A}$, pycnidia sporulation; $\mathbf{B}$, conidia production per pycnidium; and $\mathbf{C}$, conidia production per canker. Response surfaces were generated from the nonlinear form of the Richards model and associated parameter functions using coefficients derived from the pooled experimental repetition 1, 2, and 3 data sets (equation 5). Pearson correlation coefficients, $r$, were calculated to measure the degree of association between the back-transformed predictions of the model and the observed levels of sporulation. model. However, the models describing the percentage of sporulating pycnidia (S1) had one or more significantly different parameters. Although statistical comparisons of individual parameter estimates were not performed, regression results indicated that both the amplitude $(a)$ and spread $(s)$ parameters were most different (Table 1); this was also evident when comparing model plots (Fig. 2A).

Comparison of the full and reduced quadratic models (equation 2) used to estimate the Richards rate parameter resulted in a pattern similar to that observed for estimation of $K$. A significant $F$ value of $3.62(P=0.03)$ for the $\mathrm{S} 1$ dependent variable indicated that the rep $1+2$ and 3 models were significantly different. However, very low nonsignificant $F$ values of $0.85(P=0.49)$ and 0.62 $(P=0.61)$ for the S2 and S3 variables, respectively, indicated that the predicted rates of conidia production for rep 3 were statistically equivalent to those of rep $1+2$.

Unlike the two models used for estimation of the $K$ and $r$ parameters, the final sporulation models (equation 3 ) created from the two repetition data sets were found to be statistically equivalent for all three dependent variables. Insignificant $F$ values of $2.23(P=0.09), 0.72(P=0.54)$, and $0.42(P=0.74)$ were calculated for the $\mathrm{S} 1, \mathrm{~S} 2$, and $\mathrm{S} 3$ variables, respectively. In contrast to the intermediate parameter estimation models, the final sporulation models were created from much larger data sets because they contained both temperature and moisture independent variables. The larger sample size increased the degrees of freedom and, therefore, the power of the tests.

Given that the rep 3 data were statistically similar to that of rep $1+2$, particularly for the S2 and S3 variables, data from all three experimental repetitions were pooled to improve model precision. The nonlinear relationship between sporulation $(S)$, duration of high relative humidity $(H)$, and temperature $(T)$ for this reduced model can be stated as follows:

$$
S=K\left\{[1-\exp (-\rho)]^{1 /(1-m)}\right\}
$$

where $S$ represents the percentage of sporulating pycnidia (S1), the number of conidia per pycnidium (S2), or the number of conidia per canker $(\mathrm{S} 3) ; K=a \exp \left[-s(T-t)^{2}\right]$ with parameters defined in Table $1 ; \rho=b_{1} H+b_{2} H T+b_{3} H T^{2}$ with parameters defined in Table 3; and $m=0.9$. If solved for various values of $H$ and $T$, these equations describe a three-dimensional response surface depicting sporulation (Fig. 3A to C). The back-transformed predictions generated by this nonlinear model were moderately to highly correlated with observed levels of sporulation, with $r$ values ranging from 0.68 to 0.78 . Of the three dependent variables, the greatest predictive capability occurred for number of conidia per pycnidium (Fig. 3B).

\section{DISCUSSION}

Sporulation of $P$. amygdali on peach twig cankers was quantified across a wide range of temperatures and durations of high relative humidity. Optimum temperatures for cirri formation, as estimated by fitting the Gaussian model to data maxima, occurred at 19 to $20^{\circ} \mathrm{C}$. Optimum temperatures for conidia production were estimated by the model to be slightly warmer at 22 to $23^{\circ} \mathrm{C}$. Although these two sporulation processes differed in their temperature optima, cirri formation and conidia production were observed over a fairly similar and broad range of 0 to $37^{\circ} \mathrm{C}$. Regardless of the temperature, very little pycnidia sporulation and conidia production were observed during the first $8 \mathrm{~h}$ of incubation at high relative humidity. At optimum temperatures, the majority of sporulation occurred between 16 and $48 \mathrm{~h}$ after initiation of the wetting/high humidity period. Less than favorable temperatures within the 0 to $37^{\circ} \mathrm{C}$ range reduced the rate of sporulation, causing a delay in conidia production and an overall decrease in the maximum number of spores produced for any given length of incubation. 
The ability of the pathogen to sporulate over a broad range of temperatures, and in particular at cooler temperatures between 0 and $20^{\circ} \mathrm{C}$, most likely plays a significant role in the epidemiology of constriction canker. Results from a study on leaf scar infection in the fall indicated that that these natural wounds become increasingly resistant as the time interval after defoliation increases (2). Furthermore, this earlier study demonstrated that leaf scars remained susceptible for a longer period after leaf abscission at $4.4^{\circ} \mathrm{C}$ than at $26.7^{\circ} \mathrm{C}$. Essentially, cooler temperatures decreased the rate of scar healing and resistance development. Although conidia production by $P$. amygdali is below optimum at these cooler temperatures, inoculum should nevertheless be available for infection during these extended periods of leaf scar susceptibility. Temperatures in southern New Jersey during fall leaf abscission simultaneously favor slow scar healing and inoculum availability. For example, mean air temperatures during the leaf abscission period from 15 September through 30 November ranged from 2.1 to $21.6^{\circ} \mathrm{C}$ in 2001 and from 1.0 to $24.3^{\circ} \mathrm{C}$ in 2002. Although no data are available to show that this phenomenon occurs during spring, similar temperatures at that time may also reduce the rate of bud scale scar healing while simultaneously allowing inoculum production.

Moisture also plays an important role in constriction canker epidemiology. Relative humidity exceeding $90 \%$ has been shown to delay gum, suberin, and periderm formation on newly created leaf scars, while humidity at $100 \%$ altogether prevents their formation (3). Once these protective substances and tissue are formed, the ability of the pathogen to infect through the leaf scar is dramatically reduced. As with the temperature requirements for disease development, the same moisture conditions that prolong host susceptibility also promote the pathogen's ability to produce inoculum. Thus, frequent rains associated with periods of high relative humidity, which are common fall and spring weather events in the southern peach growing region of New Jersey, may well constitute critical infection periods. To substantiate this possibility, further research is needed to examine the interaction between the environmental criteria for both sporulation and infection.

The sporulation process was quantified by utilizing a growth model, the Richards model (18), in conjunction with a quadratic function and the Gaussian model to describe the rate and asymptote parameters, respectively. Although past studies have employed similar development approaches for modeling sporulation (13), infection $(1,11)$, and spore germination $(4,10,20)$, our study represents the first such effort for describing fungal sporulation of a pycnidial pathogen. In general, the derived models accurately characterized the shape or pattern of sporulation increase for each repetition of the experiment. That is, temperature optima and durations of high relative humidity required for maximum spore production were similar across each year of the study.

Although the final model validation procedure showed that data from each of the experimental repetitions were statistically similar and could be pooled, the observed differences in the amount of sporulation among years may nevertheless be biologically significant. For example, the number of conidia produced per pycnidium or per canker predicted by the repetition $1+2$ models was 2.2 to 3.1 times greater than predicted by the repetition 3 models. Thus, some other factor or factors, which were not included in the models, influenced the ability of pycnidia to sporulate from year to year. Differences in pycnidial density across years might be considered a viable cause for this variation in conidia production. Recent results have shown that $P$. amygdali pycnidia exhibit "pathogen compensation" by producing greater quantities of conidia at lower pycnidial densities (15). However, this same research showed that the compensation phenomenon resulted in an equivalent number of conidia per canker for cankers having a twofold difference in pycnidial density.

Another source of variability related to canker maturation or age may account for the apparent seasonal variation in amount of sporulation. Harvested cankers used in this study could have resulted from infections during the previous spring or fall. If a significant amount of spring infection occurred for any given year, then a greater proportion of the harvested cankers may have been younger and presumably more productive. Thus, when developing sporulation models for fungal pathogens having long-lived pycnidia, additional modeling may be needed to account for the effect of pycnidial age on productivity. Such considerations were not critical when modeling the sporulation of short-lived foliar lesions (13).

A third possible cause for seasonal variability in inoculum production may be the effect of the abiotic environment on canker growth and development prior to experimentation. Of particular interest would be any temperature effects during the previous summer. Average daily air temperatures from 1 June through 30 September were $24.7,27.8$, and $21.2^{\circ} \mathrm{C}$ for 1998,1999 , and 2000 , respectively. Maximum daily temperatures often exceeded $32^{\circ} \mathrm{C}$ in 1998 and 1999, while such highs were rare in 2000. The warmer 1998 and 1999 summers may have allowed cankers to achieve peak conidia production by fall; in vitro studies have shown optimum fungal growth at $28^{\circ} \mathrm{C}$ (N. Lalancette and $\mathrm{K}$. A. Foster, unpublished data). Conversely, the very cool 2000 summer may have slowed canker development, resulting in a greater proportion of immature, less productive cankers for the third replication. Further experimentation on the effect of temperature on fungal colonization (9) and pycnidia maturation is needed to determine the importance of this factor on inoculum production.

Usefulness of the sporulation models for predictive purposes is dependent on the pathogen's infection efficiency, as well as the probability of conidia disseminating to suitable infection courts. If relatively few conidia are necessary to initiate infection, then inoculum availability will most likely not limit epidemic develoment. Our results show that conidia production occurred over a broad temperature range and after relatively short durations of high relative humidity, conditions that occur frequently during the fall and spring periods of susceptibility. However, if $P$. amygdali has a relatively low infection efficiency on peach, as would be expected for an $r$ strategist, then the sporulation process may well limit epidemics, particularly with respect to the moisture requirement. That is, longer high humidity durations would be necessary to produce enough inoculum to obtain significant levels of infection. Under these circumstances, the model could provide useful predictions by differentiating between insufficient and favorable moisture requirements. Unfortunately, no information is currently available on the infection efficiency of $P$. amygdali on peach.

Recent research has shown that as many as eight and five fungicide applications are needed during the fall leaf drop and spring bud-break through bloom periods, respectively, to provide continuous protection from infection (16). Although a large percentage of this application cost can be economically justified by preventing yield loss (14), this form of continuous protection may not be necessary. Implementation of a forecasting system would minimize fungicide use yet maintain control, because applications would only be made when environmental criteria were favorable for disease development. Results from this experiment and prior studies indicate that the combined and simultaneous effects of temperature and moisture on infection, pathogen sporulation, and infection court susceptibility will need to be integrated for such a system to be effective. Furthermore, seasonal factors that influence the amount of conidia produced, such as the effect of the environment on pycnidia maturation, may also need to be included.

\section{ACKNOWLEDGMENTS}

This research was funded in part by the New Jersey Agricultural Experiment Station. We thank F. W. Nutter, Jr. for his critical review of the manuscript prior to submission. 


\section{LITERATURE CITED}

1. Carisse, O., and Kushalappa, A. C. 1990. Development of an infection model for Cercospora carotae on carrot based on temperature and leaf wetness duration. Phytopathology 80:1233-1238.

2. Cohoon, D. F., and Daines, R. H. 1956. Peach canker (Fusicoccum amygdali): Times and sites of infections. Plant Dis. Rep. 40:304308.

3. Daines, R. H., Cohoon, D. F., Leon, I., and Brennan, E. 1958. Control of Fusicoccum canker of peach by nutrition, defoliation, and protective fungicides. Phytopathology 48:400-407.

4. Elliot, V. J. 1988. Response models for conidiospore germination and germ tube elongation of Mycosphaerella fragariae as influenced by temperature and moisture. Phytopathology 78:645-650.

5. Guba, E. F. 1953. Large leaf spot and canker of peach caused by the fungus Fusicoccum amygdali Delacr. Plant Dis. Rep. 37:560-564.

6. Guba, E. F. 1954. Fusicoccum leaf spot and canker of peach and control. (Abstr.) Phytopathology 44:111.

7. Guba, E. F. 1958. Pertinent aspects of the infection cycle and control of the peach canker pathogen, Fusicoccum amygdali. Plant Dis. Rep. 42:481-492.

8. Haenseler, C. M., and Daines, R. H. 1941. Peach canker caused by Fusicoccum amygdali. (Abstr.) Phytopathology 31:10.

9. Hirst, J. M., and Schein, R. D. 1965. Terminology of infection processes. (Abstr.) Phytopathology 55:1157.

10. Imhoff, M. W., Main, C. E., and Leonard, K. J. 1981. Effect of temperature, dew period, and age of leaves, spores, and source of pustules on germination of bean rust urediospores. Phytopathology 71:577-583.

11. Lalancette, N., Ellis, M. A., and Madden, L. V. 1988. Development of an infection efficiency model for Plasmopara viticola on American grape based on temperature and duration of leaf wetness. Phytopathology 78:794-800
12. Lalancette, N., Foster, K. A., and Robison, D. M. 2001. Modeling sporulation of Phomopsis amygdali on peach. (Abstr.) Phytopathology 91(suppl.):S51.

13. Lalancette, N., Madden, L. V., and Ellis, M. A. 1988. A quantitative model for describing the sporulation of Plasmopara viticola on grape leaves. Phytopathology 78:1316-1321.

14. Lalancette, N., and Polk, D. F. 2000. Estimating yield and economic loss from constriction canker of peach. Plant Dis. 84:941-946.

15. Lalancette, N., and Robison, D. M. 2001. Seasonal availability of inoculum for constriction canker of peach in New Jersey. Phytopathology 91:1109-1115.

16. Lalancette, N., and Robison, D. M. 2002. Effect of fungicides, application timing, and canker removal on incidence and severity of constriction canker of peach. Plant Dis. 86:721-728.

17. Neter, J., and Wasserman, W. 1974. Applied Linear Statistical Models. Richard D. Irwin, Inc., Homewood, IL.

18. Richards, F. J. 1959. A flexible growth function for empirical use. J. Exp. Bot. 10:290-300.

19. Roberts, J. W. 1940. The constriction disease of peach. Phytopathology 30:963-968.

20. Tamm, L., and Flückiger, W. 1993. Influence of temperature and moisture on growth, spore production, and conidial germination of Monilinia laxa. Phytopathology 83:1321-1326.

21. Tuset, J. J., and Portilla, M. T. 1989. Taxonomic status of Fusicoccum amygdali and Phomopsis amygdalina. Can. J. Bot. 67:1275-1280.

22. Uddin, W., and Reynolds, K. L. 1997. Availability and dispersal of inoculum for Phomopsis shoot blight epidemics of peach. (Abstr.) Phytopathology 87(suppl.):S99.

23. Zadoks, J. C., and Schein, R. D. 1979. Epidemiology and Plant Disease Management. Oxford University Press, New York.

24. Zehr, E. I. 1995. Constriction canker. Pages 31-32 in: Compendium of Stone Fruit Diseases. J. M. Ogawa, G. W. Bird, D. F. Ritchie, K. Uriu, and J. K. Uyemoto, eds. The American Phytopathological Society, St. Paul, MN. 